\title{
ÍNDICE DE HERDABILIDADE NA ANTROPOMETRIA E LATERALIDADE DE GÊMEOS DO MUNICÍPIO DE RIO DE JANEIRO
}

\author{
Index heritability in anthropometry and laterality of gemini of \\ the rio de janeiro city
}

\author{
Glauber Lameira de Oliveira ${ }^{1,2}$; Talita Adão Perini ${ }^{2}$; Débora dos Santos Ornellas ${ }^{3}$; Paulo Moreira Silva Dantas ${ }^{4}$; \\ José Fernandes Filho ${ }^{3}$ \\ ${ }^{1}$ Instituto Federal de Educação,Ciência e Tecnologia de Rondônia, Ji-Paraná - RO - Brasil \\ 22 Centro Universitário Luterano de Ji-Paraná (CEULJI/ ULBRA) - Ji-Paraná - RO - Brasil \\ ${ }^{3}$ Universidade Federal do Rio de Janeiro, Rio de Janeiro - RJ - Brasil. \\ ${ }^{4}$ Universidade Federal do Rio Grande do Norte, Natal - RN - Brasil.
}

\begin{abstract}
Resumo: A competitividade no âmbito do esporte de alto nível tem despertado interesse por pesquisas no campo da genética. O objetivo geral deste estudo foi verificar o índice de herdabilidade nas variáveis antropométricas e lateralidade em gêmeos do município de Rio de Janeiro. Participaram da amostra 32 pares de gêmeos de ambos os sexos, sendo 23 pares monozigóticos (9 pares do sexo masculino e 14 pares do sexo feminino) e 9 pares dizigóticos (5 pares do sexo masculino e 4 pares do sexo feminino),compreendendo uma faixa etária de 7 a 40 anos de idade. Todos os gêmeos participantes eram residentes de área urbana pertencentes à capital do Estado do Rio de Janeiro, sendo estes, praticantes ou não praticantes de atividade física. A zigosidade foi obtida pelo Questionário de Peeters (1998) e a lateralidade por meio de uma anamnese de auto-relato. Para as medidas antropométricas de massa corporal total e estatura, adotou-se as padronizações internacionais da ISAK (2000). Para análise estatística aplicou-se uma análise multivariada, (técnica de Cluster analysis) e nível de significância $p<0,05$. Toda a análise estatística foi realizada no Programa Estatístico Statistical Package for Social Sciences (SPSS) for Windows versão 13.0, ano 2006. O estudo demonstrou que as variáveis determinadas essencialmente pela influência ambiental são: massa corporal total ( $\left.h^{2}=55,5 \%\right)$, índice de massa corporal $\left(h^{2}=41 \%\right)$ e lateralidade / preferência lateral $(H=0)$, diferente da estatura, determinada pela herança genética do indivíduo ( $h^{2}=$ $76,1 \%$ ). O conhecimento prévio das variáveis determinadas predominantemente por herança genética e daquelas determinadas pela influência do meio, portanto treináveis, são fundamentais, pois auxilia do processo de orientação esportiva, bem como no treinamento, possibilitando o êxito esportivo.
\end{abstract}

Palavras-chave: Gêmeos, genótipo, fenótipo.

\begin{abstract}
The competitiveness in high-level sports has attracted researchers' interest in genetics' field. The general purpose of this study was to verify the index of heritability in anthropometric variables and laterality in twins in the county of Rio de Janeiro. Thirty-two pairs of twins from both sexes took part in the sample, being 23 monozygotic pairs ( 9 male and 14 female pairs) and 9 dizygotic pairs ( 5 male and 4 female pairs), with ages ranging from 7 to 40 years-old. All twins were urban residents, living at Rio de Janeiro State Capital, being practitioners or non-practitioners of physical activity. The zygosity was obtained by the Peters' Questionnaire (1998) and the laterality by means of a self-reported anamnesis. The international standardizations from ISAK (2000) were adopted for the anthropometric measures of total body mass and height. For statistical analysis it was applied a multivariate analysis (Cluster analysis technique) and significance level $p<0.05$. All statistical analyses were performed in the Statistical Software Statistical Package for Social Sciences (SPSS), version 13.0 for Windows. The study demonstrated that the variables determined primarily by environmental influence are: total body mass $\left(h^{2}=55.5 \%\right)$, body mass index $\left(h^{2}=41 \%\right)$ and laterality/lateral preference $(H=0)$, different from height, determined by the individual's genetic inheritance $\left(h^{2}=76.1 \%\right)$. The prior knowledge of the variables predominantly determined by genetic inheritance and those determined by environmental influence, therefore trainable, are of great interest, because it can help the process of sportive orientation and training, enabling the success in sports.
\end{abstract}

Keywords: Twins, genotype, phenotype.

Aceito em 15/06/2009 - Rev. Educ. Fís. 2009 - 03-10. Rio de Janeiro - RJ - Brasil

\section{INTRODUÇÃO}

Atualmente têm-se desenvolvido pesquisas na área da genética atreladas ao âmbito da atividade física para fins de orientação esportiva, a partir do conhecimento da relação existente entre fatores genotípicos (intrínsecos)eambientais (extrínsecos) na determinação de características fenotípicas de indivíduos ${ }^{(1,2,3)}$. Tais informações têm despertado interesse da comunidade científica, evocando a 
necessidade de realizar discussões aprofundadas por parte dos profissionais que militam na área da atividade física no que concerne às características físicas e aptidões de determinado individuo (4).

Há mais de duas décadas, pesquisadores de diferentes áreas das ciências do desporto tentam identificar os fatores que podem influenciar o rendimento esportivo de um indivíduo, a fim de determinar o prognóstico do desempenho esportivo do mesmo $(5,6,7)$. Este conhecimento possibilita intervenções no que tange ao treinamento, na orientação para a iniciação esportiva e direcionamento de atletas a determinadas modalidades esportivas, a fim de atentar para as reais capacidades físicas, que o individuo, a partir da interação e exposição ao meio, pode vir a desenvolver. Haja vista, que a partir do conhecimento de quanto mais baixo for o impacto da genética sobre uma dada característica, mais treinável será esta característica ${ }^{(8)}$.

Segundo Beunen e Thomis ${ }^{(9)}$, o fator genético e as condições ambientais adequadas são précondições esportivas que propiciam ao atleta desenvolver bem as habilidades necessárias para a prática do esporte . Esta idéia sugere, que a "excelência" em qualquer modalidade esportiva depende do treinamento associado às características genéticas ${ }^{(10)}$.

As particularidades biológicas e ambientais de um indivíduo têm sido estudadas por métodos que avaliam as características de caráter hereditário (7). O método de estudos em gêmeos possibilita observar o grau de influência da hereditariedade e do ambiente. Caso a variável a ser estudado do organismo for dependente da hereditariedade, os homozigotos serão muito semelhantes. Por outro lado, quanto mais as vaiáveis dependam do meio ambiente, maior será a diferença entre eles. Este fato ocorre, porque os gêmeos monozigóticos possuem genótipos iguais e qualquer diferença entre eles será resultado da influência do meio ambiente (2). Logo, as diferenças observadas entre esta classe de gêmeos acontecem quando estes são educados em condições diferentes ou submetidos a fatores diferentes de treinamento (11). Este conhecimento sucinta a necessidade de investigar o grau de influência genotípica e ambiental na determinação de variáveis morfofuncionais.

Portanto, o objetivo geral deste estudo foi verificar o índice de herdabilidade nas variáveis antropométricas e na lateralidade em gêmeos do município de Rio de Janeiro.

\section{METODOLOGIA}

\section{Amostra}

Participaram deste estudo um total de 32 pares de gêmeos de ambos os sexos (64 pessoas), sendo 23 pares monozigóticos ( 9 pares do sexo masculino e 14 pares do sexo feminino) e 9 pares dizigóticos (5 pares do sexo masculino e 4 pares do sexo feminino), com idade entre 7 e 40 anos, residentes do município do Rio de Janeiro.

Todos os participantes do estudo assinaram um termo de consentimento livre e esclarecido devidamente aprovado pelo Comitê de Ética em Pesquisa (COMEP) da Universidade Castelo Branco com o protocolo de $\mathrm{n}^{\circ}$ 0009/2008, concordando com os procedimentos adotados durante a coleta de dados.Quando menores de idade foi solicitado à autorização prévia dos pais, que foram igualmente informados de todos os procedimentos adotados pelo estudo. O devido Termo, obedeceu ao prescrito na Resolução 196/96 de 10 de outubro de 1996, sobre as Diretrizes de Normas Regulamentares de Pesquisas Envolvendo Seres Humanos, do Conselho Nacional de Saúde.

\section{Determinação da zigotia e lateralidade}

1. Anamnese: instrumento de auto-relato, previamente validado, aplicado para a determinação da predominância lateral, bem como para registro de dados antropométricos obtidos pela avaliação antropométrica ${ }^{(2)}$.

2. Questionário de Zigotia de Peeters: Para a determinação da zigotia entre os gêmeos optouse por utilizar um método indireto, o questionário proposto por Peeters et al ${ }^{(12)}$, uma vez que este apresenta elevada validade (98\%) frente aos métodos de determinação direta da zigosidade como o DNA e os marcadores sanguíneos, tal como confirma o estudo de Maia et al., ${ }^{(5)}$. Ressaltase que no presente estudo não foi determinada a validade e fidedignidade do procedimento de Peeters.

A fim de garantir os mesmos procedimentos de coleta dos dados, para todos os pares de gêmeos, optou-se pela realização da seguinte seqüência: $1^{\circ}$ Aplicação do questionário de zigosidade; $2^{\circ}$ 
Aplicação da anamnese para identificação da preferência lateral e $3^{\circ}$ Avaliação antropométrica.

\section{Avaliação antropométrica}

Todos os participantes foram submetidos à coleta de dados antropométricos de estatura e massa corporal total. Para a medida de estatura utilizou-se um estadiômetro (WCS, ano 2006, provido de uma escala métrica graduada em centímetros). Para a Massa Corporal Total utilizou-se uma balança digital (FILIZOLA , ano 2003 com precisão de 100 gramas). Todas as medidas tomadas seguiram as padronizações da Sociedade Internacional para o Progresso da Cineantropometria (International Society for Advancement in Kinanthropometry ISAK) ${ }^{(13)}$. Os avaliadores foram submetidos a um treinamento prévio para aplicação dos questionários e padronização da tomada de medidas antropométricas. obtendo-se nesta última, o erro técnico de medida antropométrica aceitável, garantindo-se a fidedignidade da pesquisa ${ }^{(14)}$. A partir das medidas obtidas, foi calculado o Índice de Massa Corporal (Kg/m2).

\section{Estimativa do índice de herdabilidade}

A estimativa do Índice de Herdabilidade $\left(\mathrm{h}^{2}\right)$ consiste na comparação de diferenças ao nível de um dado caráter observado em gêmeos monozigóticos (MZ) e dizigóticos (DZ).

Para caracteres de variação quantitativa, utilizam-se os coeficientes de correlação obtidos entre os valores das séries de gêmeos $M Z$, por um lado, e de gêmeos DZ, por outro:

$h^{2}=\frac{r M Z-r D Z}{1-r_{D Z}}$

Onde:

$r \mathrm{MZ}=$ correlação nos pares monozigóticos

r DZ= correlação nos pares dizigóticos

Em relação aos caracteres de caráter qualitativo aplica-se a equação abaixo:

$H=\frac{C_{M Z}-C_{D Z}}{1-C_{D Z}}$

Onde:

$\mathrm{CMZ}$ = concordância nos pares monozigóticos

CDZ= Concordância nos pares dizigóticos
Quando $\mathrm{h}^{2}=1 / \mathrm{H}=1 \quad(100 \%)$, o caráter observado entre os gêmeos é atribuível exclusivamente a causas genéticas, já que os gêmeos $M Z$ são concordantes. Logo, têm-se que $\mathrm{H}$ e $\mathrm{h}^{2}$ será tanto maior quanto menor for a participação do ambiente na sua determinação ${ }^{(15)}$. Porém, se $\mathrm{h}^{2}=0 / \mathrm{H}=0$, o caráter observado entre os gêmeos é explicado pelos fatores ambientais.

\section{Análise estatística}

O presente estudo observou dois momentos distintos na composição do Tratamento Estatístico, o primeiro relativo à análise descritiva dos dados, segundo os parâmetros de média e desvio-padrão para descrição das variáveis. O segundo, no sentido de avaliarmos de modo diferenciado os índices de herdabilidade calculados de acordo com as variáveis experimentais, aplicou-se uma Análise Multivariada, a Técnica de segmentação por Agrupamentos (Cluster analysis) observando a distância Euclidiana e como Método de Partição - método heurístico K-médias combinada a correlação de Pearson como medida de proximidade, tendo em vista segmentar as variáveis de acordo com os respectivos níveis de herdabilidade, denotando grupos distintos ordenados segundo uma escala crescente destes níveis: herdabilidade baixa $\left(h^{2}<60 \%\right)$, herdabilidade intermediária $\left(60 \% \leq \mathrm{h}^{2}<80 \%\right)$ e herdabilidade alta ( $80 \% \leq \mathrm{h} 2 \leq 100 \%$ ).

O número de grupos foi definido a priori, a Análise de Variância One way como método de ratificação das diferenças inter grupos e como referência para crítica da Hipótese Nula um nível de significância $p<0,05$. Toda a análise estatística foi realizada no Programa Estatístico Statistical Package for Social Sciences (SPSS) for Windows versão 13.0, ano 2006.

\section{RESULTADOS}

\section{Características antropométricas}

A TABELA 1, abaixo, é representativa do Índice de Herdabilidade nas variáveis antropométricas (natureza quantitativa) de todos os pares de gêmeos monozigotos e dizigotos participantes da amostra deste estudo. Observa-se que o índice de herdabilidade foi elevado para a variável estatura e baixo para a massa corporal e IMC nos grupos sob análise. 
TABELA 1

ÍNDICE DE HERDABILIDADE NA

ANTROPOMETRIA

\begin{tabular}{lrrr}
\hline & & & $\begin{array}{c}\text { ÍNDICE } \\
\text { VARIAVEL }\end{array}$ \\
& MONOZIGOTO & DIZIGOTO & HERDABILIDADE \\
& Irmão 1 Irmão 2 & Irmão 1 Irmão 2 & $h^{2}$ \\
\hline
\end{tabular}

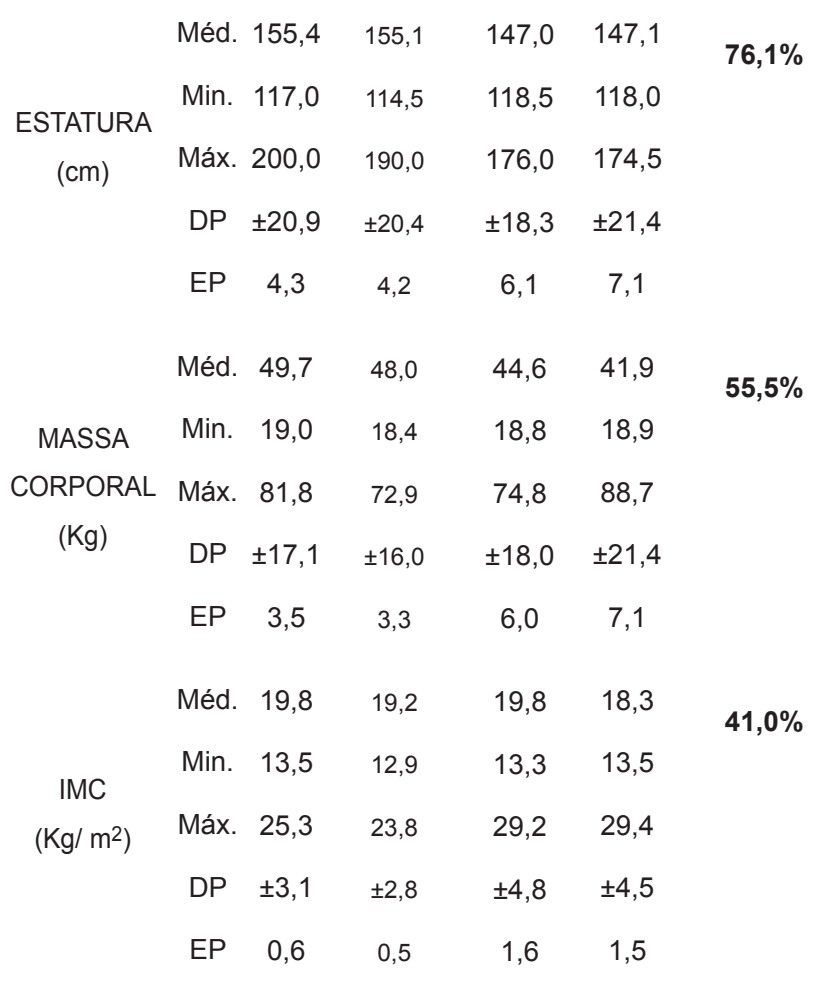

Valores em média(Méd), mínimo (Min),máximo (Max), desvio padrão(DP), Erro padrão(EP) e índice de herdabilidade $\left(\mathrm{h}^{2}\right)$ para antropometria.

\section{Lateralidade}

Na TABELA 2, ao lado, são dispostos os valores da variável lateralidade (natureza qualitativa), seu respectivo índice de herdabilidade e concordância entre os gêmeos monozigóticos e dizigóticos.

A concordância para a lateralidade, apresentada entre os dizigóticos foi maior do que a apresentada pelos monozogóticos, explicitando a influência ambiental na determinação desta variável. Da mesma forma, a amostra não apresentou herdabilidade para esta variável, resultante da ausência da influência hereditária na sua determinação.
TABELA 2

ÍNDICE DE HERDABILIDADE NA LATERALIDADE

\begin{tabular}{|c|c|c|c|}
\hline \multirow{2}{*}{ VARIAVEL } & \multicolumn{2}{|c|}{ CONCORDÂNCIA } & \multirow{2}{*}{$\begin{array}{c}\text { HERDABILIDADE }(\mathrm{H}) \\
\text { Todos }\end{array}$} \\
\hline & $M Z$ & $\mathrm{DZ}$ & \\
\hline LATERALIDADE & $82,6 \%$ & $88,9 \%$ & (7 a 40 anos) \\
\hline
\end{tabular}

\section{DISCUSSÃO}

No presente estudo, ressalta-se que a discrepância observada entre as faixas etárias (7 a 40 anos), evidenciada pela amplitude do desvio padrão das variáveis antropométricas (TABELA1), não implica influência na determinação do valor do índice de herdabilidade das variáveis avaliadas, foco deste estudo. Haja vista, que tal índice $\left(h^{2}\right)$ é determinado a partir da correlação de uma dada variável, obtida entre o irmão 1 e 2 de cada par de gêmeos (MZ ou DZ) independentemente, cujas idades são as mesmas. Outros estudos de mesmo cunho, igualmente apresentaram elevada discrepância de idades em suas amostras, como evidenciado nas pesquisas realizadas por Arya et al., (16) e Kalichman et al., (17), com variabilidade de faixa etária de 6 a 72 anos e de 18 a 90 anos, respectivamente.

$\mathrm{Na}$ antropometria, o baixo índice de herdabilidade $\left(h^{2}<60 \%\right)$ apresentado pelas variáveis de massa corporal total e índice de massa corporal, denota que este último, uma vez obtido pela razão entre a massa corporal total pela estatura ao quadrado, sofre grande influência ambiental na sua determinação $\left(h^{2}=\right.$ $59 \%$ ) em função da interferência mais expressiva da massa corporal do indivíduo. Haja vista, que a estatura, outro componente da equação, possui herdabilidade intermediária $60 \% \leq \mathrm{h}^{2}<80 \%$ ) e, por isso, é determinada prevalentemente pelo caráter genético. Estes resultados podem ser corroborados por Fernandes Filho e Carvalho ${ }^{(8)}$ e Skinner ${ }^{(18)}$. Este último afirma, a partir de estudos comparativos em gêmeos monozigóticos realizados em diferentes populações, que a 
herança genética tem alto impacto sobre a variável antropométrica estatura , o que é ratificado por Katzmarzyk et al., ${ }^{(19)}$ e Gaist et al., ${ }^{(20)}$. Fernandes Filho \& Carvalho ${ }^{(8)}$ sugerem, em seu estudo, que há $98 \%$ de influência da hereditariedade sobre a estatura, ratificando a prevalência genética na sua determinação. Igualmente, Rice et al., (21) constataram a grande influência que o componente genético tem sobre a estatura corporal. Estudos recentes realizados por Reis et al., ${ }^{(22)}$ e Silvertonen et al., ${ }^{(23)}$ obtiveram valores de h2 $=95 \%$ e $h^{2}=77 \%$ a $96 \%$, respectivamente para estatura, o que confirma tal influência.

Em uma pesquisa realizada por Klump et al., (24) com gêmeos monozigóticos que cresceram sob condições ambientais iguais e gêmeos monozigóticos que cresceram em condições diferentes (alimentação, educação, e outros) comprovou que os gêmeos apresentaram alta similaridade das características físicas, precisamente para a variável IMC quando criados sob os mesmos estímulos externos, porém aqueles submetidos a estímulos ambientais diferentes, apresentam diferença para esta variável em razão de apresentarem diferenças para a massa corporal. A similaridade das características físicas aumenta quando os gêmeos compartilham das mesmas atividades (instituição educacional, tarefas diárias, eventos sociais, prática de atividades físicas) ${ }^{(25)}$. Esta última informação sugere a influência dos fatores ambientais sobre a predisposição genética nas variáveis de massa corporal e IMC, corroborando com o presente estudo.

A massa corporal apresentou baixa herdabilidade $\left(h^{2}=55,5 \%\right)$ e, portanto, alta influência ambiental na sua determinação. De acordo com este estudo, pesquisas comprovam ${ }^{(6,22)}$, que o caráter hereditário não é determinante na constituição dessa massa corporal. Reis et al., (22), apresentaram em seu estudo, valor de $h^{2}=$ $52 \%$ para esta variável, evidenciando a influência ambiental, como no presente estudo.Logo, o estilo de vida, precisamente o aspecto nutricional, é preponderante ao ganho ou perda de massa corporal total. Os resultados apresentados na TABELA 1 coincidem com os encontrados em um estudo realizado por Kaplowitz et al., (26). De igual modo, este estudo mostrou que os padrões de massa corporal apresentam-se com uma baixa herdabilidade, exercendo apenas $38 \%$ de influência genotípica sobre esta variável, apresentando $\mathrm{h}^{2}=62 \%$.

O presente estudo apresentou baixa influência genotípica para a variável IMC, já que neste a herdabilidade foi de $41 \%$ (TABELA 1). De igual modo, Pietilainen et al., (27), realizou um estudo com pares de gêmeos de 20 a 25 anos com o objetivo de observar a herdabilidade no índice de massa corporal (IMC) entre eles, e comprovou que a influência ambiental foi maior que a genética.

A maioria dos estudos estimam uma baixa herdabilidade para o IMC, com valores entre 40 - 55\% (6, 21), próximos ao deste estudo, representando alta influência ambiental na sua determinação.

Estes dados estão em acordo com estudos prévios que mostram a preponderância de fatores ambientais no desenvolvimento de certas características físicas, precisamente IMC e massa corporal total ${ }^{(21,28,29)}$.

Para análise da lateralidade, observou-se que foram obtidos valores mais elevados para os graus de concordância entre gêmeos dizigóticos em comparação com gêmeos monozigóticos. Este resultado vai de encontro à hipótese de que irmãos com a mesma carga genética apresentam necessariamente as mesmas características fenotípicas de simetria lateral ou assimetria lateral direita ou esquerda.

Quanto à lateralidade, não foram obtidos valoresdeherdabilidade paraosgêmeos avaliados. Para ${ }^{(30)}$ e ${ }^{(31)}$, a ausência de herdabilidade indica que a variância genética não contribui para as diferenças de lateralidade observadas entre os indivíduos, o que corrobora com os resultados já apresentados, reforçando a premissa de que o ambiente exerce fundamentalmente influência na determinação da lateralidade, ou seja, simetria ou assimetria direita ou esquerda. Um estudo recente desenvolvido por Kalichman et al., ${ }^{(17)}$ apresentou na sua amostragem um valor de herdabilidade de $h^{2}=19 \%$ para esta variável.

Jancke e Steinmetz ${ }^{\left({ }^{32}\right)}$ desenvolveram um estudo com pares de gêmeos monozigóticos concordantes destros ou discordantes em lateralidade. Os autores mensuraram o desempenho máximo com as mãos direita e esquerda em tarefas de velocidade de toques repetidos e de escrita. O estudo comprovou 
que, apesar de possuírem heranças genéticas iguais, as assimetrias laterais observadas em cada sujeito não prediziam o grau de assimetria lateral e o desempenho apresentado pelo gêmeo correspondente.

Por outro lado, fatores ambientais têm demonstrado possuir um papel importante na determinação de diferenças laterais de desempenho, com particular destaque para 0 efeito da quantidade de prática específica em cada membro (32). Desta forma, os resultados deste estudo suportam a proposição de que as assimetrias laterais podem ser um fator específico à tarefa, em função do seu caráter não determinístico pela herança genética, e de seu caráter mutável como conseqüência de fatores ambientais interferindo nas funções motoras simples ou complexas que exercem.

No âmbito esportivo, a partir do conhecimento apresentado neste estudo referente à lateralidade, será possível proporcionar ao atleta certas vantagens estratégicas a partir do treinamento de ambos os segmentos corporais (direito e esquerdo), com vistas a desenvolver a simetria das ações motoras em um dado atleta, proporcionando uma maior possibilidade de atuação, a partir da variabilidade de execução de gestos esportivos. Um estudo desenvolvido por Cardoso ${ }^{(33)}$, objetivou promover uma reorganização psicomotora por meio do desenvolvimento da lateralidade ou simetria dos gestos motores em crianças de até 12 anos. O estudo comprovou que após o período de cinco meses de treino, os avaliados apresentaram desenvolvimento da lateralidade, passando a apresentarem simetria lateral.

No esporte de alto nível, alguns estudos (11, 32) têm incidido na importância que um tipo de execução simétrica nas habilidades específicas esportivas podem ter para um conseqüente êxito na manifestação competitiva de alto rendimento. Neste sentido, a simetria das habilidades esportivas poderia ser um diferencial do desempenho esportivo bem sucedido.

Diversos trabalhos ${ }^{(32,34,35)}$ parecem indicar a extraordinária importância que as manifestações de lateralidade supõem na intervenção esportiva, por isso, se faz necessário se aprofundar nas possibilidades que oferece a utilização de outra preferência lateral, a partir do desenvolvimento da simetria lateral, característica apontada pelo estudo como capaz de ser treinável, e, portanto, contribuir para o êxito esportivo.

Para Fernandes Filho e Carvalho ${ }^{(8)}$ o nível contemporâneo dos êxitos desportivos que visa a grandes exigências do organismo do desportista, faz suscitar a necessidade de existir um treinamento esportivo eficaz, capaz de condicionar o atleta ao seu máximo desempenho.

Por meio de estudos como este, é possível contribuir com mais um recurso a ser adicionado no âmbito do esporte, cuja meta é atender toda a potencialidade do atleta.

O fato o presente estudo não ter controlado o nível de atividade física, bem como padrão alimentar dos voluntários pode-se considerar como um fator limitante do estudo, sugerindo que esta medida seja adotada em estudos posteriores.

\section{CONCLUSÃO}

O estudo constatou que as variáveis antropométricas de índice de massa corporal e massa corporalsão determinadasprevalentemente por fatores ambientais, enquanto que a estatura apresenta forte influência genética. Quanto à lateralidade, foi verificado que esta apresentou forte influência ambiental na sua determinação, comportando-se como uma habilidade motora treinável.

Recomenda-se que a partir do presente estudo, profissionais que militam a área da Atividade Física e Desportos possam aplicar em sua prática profissional um novo recurso adicional ao programa de treinamento, aumentando a performance atlética e otimizando o processo de orientação esportiva, considerando as potencialidades capazes de serem desenvolvidas, portanto treináveis (influenciáveis pelo meio).

Sugere-se a aplicabilidade da herdabilidade em estudos futuros, incluindo um maior número de pares de gêmeos e a investigação de outras variáveis morfofuncionais que possam corroborar a orientação esportiva eficiente.

\section{REFERÊNCIAS BIBLIOGRÁFICAS}

1. Bayoumi RA, AL-Yahyaee AS. Albarwani SA, Rizvi SG, Al-Hadabi S. Herdability of determinants of metabolic syndrome among healthy Arabs of the Oman family study. Obesity 2007; 15,3: 551-6. 
2. Machado JFV. Análise da Influência genotípica e do ambiente sobre a dermatoglifia, o somatotipo, as qualidades físicas e a maturação sexual em gêmeos. Rio de Janeiro, 2005: 202p, Dissertação (Mestrado em Ciências da Motricidade Humana), Universidade Castelo Branco.

3. Silva Dantas PM, Fernandes Filho J. Identificação dos perfis, genético, de aptidão física e do somatotipo que caracterizam atletas masculinos, de alto rendimento, participantes do futsal adulto, no Brasil. Fitness \& Performance Journal 2002; 1,1:28-36.

4. Silva Dantas PM, Alonso L. Futsal e Dermatoglifia. In: Dantas EHM, Fernandes Filho J, editors. Atividade física em ciências da saúde. 1 ed. Rio de Janeiro: editora Shape, 2005.

5. Maia JAR, Fernandes SCT, Amorim A, Alves C, Gusmão L, Pereira L. Determinação da gemelaridade do questionário de Peeters aos micro-satélites aleatórios espalhados pelo DNA. Rev Port Cien Desp 2007;7,2:147-155.

6. Bouchard C,Tremblay A. Genetic influences on the response of body fat and fat distribution to positive and negative energy balances in human identical twins. J Nutr, 127(5 Suppl): 943S-947S, 1997.

7. Feitosa MF, Krieger H. O futuro da epidemiologia genética de características complexas. Cien Saude Colet 2002; 7,1:73-83.

8. Fernandes Filho J, Carvalho JLT. Potencialidades desportivas de crianças segundo a perspectiva da escola soviética. Rev. Bras. Cineantropom. Desempenho Hum 1999; 1,1: 96-107.

9. Beunen G, Thomis M. Genetic determinants of sports participation and daily physical activity. Int J Obes Relat Metab Disord, 23 Suppl 3: S55-63, 1999.

10. Filin \& Volkov, V. Seleção de Talentos nos Desportos. Londrina: Midiograf,1998.

11. Anokhin AP, Heath A, Myers E. Genetic and environmental influences on frontal eeg asymmetry: a twin study. Bil Psichols 2006; 71,3: 289-95.

12. Peeters H, Van Gestel S, Vlietinck R, Derom C, Derom, R. Validation of a telephone zigosity questionnaire in twins of known zygosity. Behavioral Genetics 1998;28,1:159-163.

13. Norton K, Olds T. Antropometrica. Biosystem, servicio educativo, Rosário, Argentina, 2000.

14. Perini TA, Oliveira GL, Ornellas JS, Oliveira FP. Cálculo do erro técnico de medição em antropometria. Rev Bras Med Esporte 2005; 11,1:81-90.
15. Beiguelman B. Dinâmica dos genes nas famílias e nas populações. 1 ed. Ribeirão Preto: Sociedade Brasileira de Genética, 1994.

16. Arya R, Duggirala R, Comuzzier AG, Puppala S, Modem S, Busi BR, et al. Reritability of anthropometric phenitypes in caste populations os Visaklapatnam, Índia. Hum Biol, 2002; 74, 3, 325-44.

17. Kalichman L, Korostishevsky M, Kobyliansky E. Laterality índices the Chuvashian population. Anthropol Anz 2008; 66,4:409-18.

18. Skiner JS. Do genes determine champions? Gatorade sports science institute sports science exchance 2001;14,1: 1-4.

19. Katzmarzyk PT, Malina RM, Perusse L, Rice T, Province MA, Rao DC. Familial resembrance in fatness and fat distribution. Am J Human Biol 2000; 12,3: 395-404.

20. Gaist D, Bathun L, Skytthe A, Jersen TK, Mcgue M, VaupeL JW .Strength and anthropometric measures in identical and fraternal twins: no evidence of masculinization of females with males co-twins. Epidemiology 2000; 11,1: 340-343.

21. Rice T, Perisse L, Bouchard C, RAO DC. Familial aggregation of by massa index and subcutaneous fat measures in the longitudinal Quebec family study. Genet Epidemiol 1999; 16,3: 316-34.

22. Reis VM, Machado JV, Fortes M, Fernandes PR, Silva AJ, Dantas PD, et al. Evidence for Heritability of somatotypr compared to body mass index in female twins. Journal physiological anthropology 2007; 26 : 9-14.

23. Silventoinen K, Bartels M, Posthuma D, Estougievan Burk GF, Willemsen G, Van Beijsterveldt TC, et al. Genetic regulation of growth in height and weight from 3 to 12 years of age: a longitudinal study of Dutch twin children. Twin Res Hum Genet 2007;10:2,354-63.

24. Klump KL, Holly A. Physical similarity and twin resemblance for eating attitudes and behaviors: a test of the equal environments assumption. Behav Genet 2000; 30,1: 51-8.

25. Lima CP .Genética Humana, 3 ed. São Paulo: Harbra, 1996.

26. Kaplowitz HJ, Wild KA. Serial and parent-child changes in components of body fat distribution and fatness in children from the London Longitudinal Growth Study, ages two to eighteen years. Hum Biol 1988; 60,5: 739-58. 
27. Pietilainen $\mathrm{KH}$, Bergholm R, Rissanen A, Kaprio J. Effects os accquired obesity on endothelial function in monozygotic twins. Obesity Research $2005 ; 14,1: 826-37$.

28. Kaprio J, Pukkinen L, Rose RJ Genetic and environmental factors in health-related behaviors: studies on finnish twins and twin families. Twin Rev 2002;5,5: 366-71.

29. Hjelmborg JVB, Fagnani C, Silventonen K. Genetic influences on growth traits of BMI: A longitudinal study of adult twins. Obesity 2008; 10,1:12-17.

30. Frederiksen, $\mathrm{H}$ e Christensen, $\mathrm{K}$. The influence of genetic factors on physical functioning and exercise in second half of life. Scandinavan Jopurnal Medicine \& Science in Sports 2002;13:9-18.

31. Sobral, F. O adolescente atleta. Lisboa: Livros Horiozonte, 1988.

32. Jancke L, Steinmetz H. Hand motor performance and degree of asymmetry in monozigotic twins. Cortex 1995; 31:799-85.
33. Cardoso SS, Almeida MCR. Efeitos de um programa de reeducação psicomotora desenvolvido para crianças com relação aos fatores psicomotores: noção de corpo e lateralidade. Movimentum 2007; 2,2:1- 13.

34. Teixeira LA, Paroli R. Assimetrias laterais em ações motoras: Preferência versus desempenho. Motriz 2000; 6,2:1-8.

35. Magalhães AF. Lateralidade: implicações no desenvolvimento infantil. 1 ed. Rio de Janeiro: Sprint, 2001.

Endereço: Rua Rio Amazonas, $\mathrm{n}^{\circ}$. 151, - Jardim dos Migrantes-Ji-Paraná-RO. cep: 78960-000 Tels.:

RESIDENCIAL: (69) 31463147

CELULAR: (69) 8441-3878

e-mail: glauberlaoli@ig.com.br 